\title{
ON MANIFOLDS WITH NONNEGATIVE CURVATURE ON TOTALLY ISOTROPIC 2-PLANES
}

\author{
WALTER SEAMAN
}

\begin{abstract}
We prove that a compact orientable $2 n$-dimensional Riemannian manifold, with second Betti number nonzero, nonnegative curvature on totally isotropic 2-planes, and satisfying a positivity-type condition at one point, is necessarily Kähler, with second Betti number 1 . Using the methods of Siu and Yau, we prove that if the positivity condition is satisfied at every point, then the manifold is biholomorphic to complex projective space.
\end{abstract}

\section{INTRODUCTION}

Let $V$ denote a real vector space with inner product $\left(\right.$, ), let $V^{\mathbb{C}}=$ $V \otimes \mathbb{C}$ denote the complexification of $V$. Extend $($,$) to a complex b i$ linear form on $V^{\mathbb{C}}$, and let $\langle\langle\rangle$,$\rangle denote the extension of ($,$) to V^{\mathbb{C}}$ which is complex linear in the first argument and conjugate linear in the second, that is $\langle\langle z, w\rangle\rangle=(z, \bar{w})$ for $z, w \in V^{\mathbb{C}}$. By an algebraic curvature tensor $R$ on $V$ we mean a multilinear map $R: V \times V \times V \rightarrow V$ satisfying (a) $R(X, Y) Z=-R(Y, X) Z$, (b) $R(X, Y) Z=R(X, Z) Y+R(Z, Y) X$ (the Bianchi identity), and (c) $(R(X, Y) Z, W)=(R(Z, W) X, Y)$. The curvature operator (of $R$ ) is defined to be the map $\widehat{R}: \Lambda^{2} V \rightarrow \Lambda^{2} V$ given by $(\widehat{R}(x \wedge y), u \wedge v)=(R(x, y) v, u)$ where $x, y, u, v \in V$ and the inner product $\Lambda^{2} V$ is given by $(x \wedge y, u \wedge v)=(x, u)(y, v)-(x, v)(y, u)$. We extend $\widehat{R}$ by $\mathbb{C}$-linearity to $\Lambda^{2} V^{\mathbb{C}}$. A (complex) vector $z \in V^{\mathbb{C}}$ is said to be isotropic if $(z, z)=0$. A complex subspace $W$ of $V^{\mathbb{C}}$ is said to be totally isotropic if $\forall z \in W, z$ is isotropic. If $z$ and $w$ are vectors in $V^{\mathbb{C}}$, then the number $\langle\langle\widehat{R}(z \wedge w), z \wedge w\rangle\rangle /\|z \wedge w\|^{2}$ depends only on the complex subspace $\sigma$ spanned by $z$ and $w$. We denote this number by $K(\sigma)$. The following definition was introduced in $[\mathrm{MM}]$.

Definition. The curvature tensor $R$ is said to have nonnegative (positive) curvature on totally isotropic 2-planes if $K(\sigma) \geq 0 \quad(>0)$ for all totally isotropic (complex) two-dimensional subspaces of $V^{\mathbb{C}}$.

Every two-dimensional totally isotropic subspace $\sigma$ of $V^{\mathbb{C}}$ has a basis of the form $\{z, w\}$, where $z=e_{i}+l e_{j}$ (the iota means $(-1)^{1 / 2}$ ), $w=e_{k}+l e_{l}$, where $e_{i}, e_{j}, e_{k}$, and $e_{l}$ denote orthonormal vectors in $V$, and conversely any

\footnotetext{
Received by the editors December 19, 1990 and, in revised form, April 19, 1991 and May 19, 1991.

1991 Mathematics Subject Classification. Primary 53C20; Secondary 53C55, 32C17.
} 
such $z$ and $w$ will span a totally isotropic 2-plane in $V^{\mathbb{C}}$. Using this choice of $z$ and $w$, one computes

$$
\langle\langle\widehat{R}(z \wedge w), z \wedge w\rangle\rangle=K_{i k}+K_{i l}+K_{j k}+K_{j l}+2 R_{i j k l}
$$

where $R_{i j k l}=\left(R\left(e_{i}, e_{j}\right) e_{k}, e_{l}\right)$ and $K_{a b}=\left(R\left(e_{a}, e_{b}\right) e_{b}, e_{a}\right)=$ the sectional curvature of the plane (in $V$ ) spanned by the orthonormal vectors $e_{a}$ and $e_{b}$. Now notice that the plane spanned by the complex conjugate, $\bar{z}=e_{i}-l e_{j}$, of the above $z$ and the above $w=e_{k}+l e_{l}$, is also a totally isotropic 2-plane. Therefore if $R$ has nonnegative curvature on (all) totally isotropic 2-planes we see from $(0.1)$ that the following must be true for all orthonormal $e_{i}, e_{j}, e_{k}, e_{l}$ :

$$
K_{i k}+K_{i l}+K_{j k}+K_{j l} \geq 2\left|R_{i j k l}\right| \text {. }
$$

An algebraic curvature tensor will have nonnegative curvature on totally isotropic 2-planes if it satisfies either of the following conditions: (i) $\widehat{R}$ is a nonnegative operator on $\bigwedge^{2} V\left(\widehat{R}\right.$ is symmetric on $\left.\bigwedge^{2} V\right)$. (ii) The sectional curvature of $R$ is quarter-pinched (i.e., for every two-dimensional subspace $P$ of $V, 4 \geq K(P) \geq 1)$. Thus nonnegative curvature on totally isotropic 2-planes is implied by conditions which are familiar to geometers. A Riemannian manifold $M$ is said to have nonnegative (positive) curvature on totally isotropic 2-planes if its Riemannian curvature satisfies the corresponding property on each tangent space. In particular, every Riemannian symmetric space of compact type has nonnegative curvature on totally isotropic 2-planes owing to (i). This apparently weak condition sees to be "exactly right" for controlling the behavior of stable harmonic maps from the 2-sphere $S^{2}$ into $M$. The authors of $[\mathrm{MM}]$ demonstrate the power of this notion in proving the following remarkable

Theorem. Let $M$ be a compact simply connected $n$-dimensional Riemannian manifold which has positive curvature on totally isotropic 2-planes, where $n \geq 4$. Then $M$ is homeomorphic to a sphere.

Note that as a corollary to this theorem, any compact simply connected Riemannian manifold whose sectional curvature is pointwise strictly quarterpinched (that is, there is a positive function $f$ on $M$ such that at any point $p \in M$, the sectional curvature of $M$ at $p, K(p)$, satisfies $4 f(p) \geq K(p)>$ $f(p))$ must be homeomorphic to a sphere. This represents an important generalization (in our opinion) of that portion of the "classical" Sphere Theorem which states that if $M$ is globally strictly quarter-pinched $(4 \geq K>1)$ then $M$ is homeomorphic to a sphere.

Let $(*)$ denote the following condition for an algebraic curvature tensor:

(*) For all orthonormal vectors $e_{i}, e_{j}, e_{k}, e_{l}, K_{i k}+K_{i l}+K_{j k}+K_{j l}>0$.

The sum in $(*)$ above may be written

$$
\frac{1}{2}(\langle\langle\widehat{R}(z \wedge w), z \wedge w\rangle)+\langle\langle\widehat{R}(z \wedge \bar{w}), z \wedge \bar{w}\rangle\rangle)
$$

where $z$ and $w$ were defined above (one could use $\bar{z} \wedge w$ in place of $z \wedge \bar{w}$ ). We shall give different interpretations of the sum appearing in $(*)$ later in this section, but for now notice that if $R$ has nonnegative curvature on totally isotropic 2-planes the sum in $(*)$ is automatically nonnegative. The purpose of this paper is to prove the following results: 
Theorem A. Let $\left(M^{2 n}, g\right), n \geq 2$, be a compact orientable $2 n$-dimensional manifold with Riemannian metric $g$. Suppose the second real Betti number of $M, b_{2}(M ; \mathbb{R})$, is nonzero. Suppose the Riemannian metric $g$ has nonnegative curvature on totally isotropic 2-planes and satisfies (*) at one point. Then (i) in a natural way, $(M, g)$ is a Kähler manifold, (ii) $b_{1}(M ; \mathbb{R})=1$, and (iii) $M$ is simply connected.

Theorem B. Let $(M, g)$ satisfy the same hypotheses as in $\underline{A}$, but now assume in addition that $(*)$ is satisfied at every point of $M$. (i) If $g$ is Einstein then $(M, g)$ is biholomorphically isometric to $\mathbb{C P}^{n}$ with a multiple of the canonical metric. (ii) In general $M$ is biholomorphic to $\mathbb{C H}^{: n}$.

The ideas behind the proofs of Theorems A and B will now be discussed. Precise definitions and statements will follow in the next section. It turns out that for an even-dimensional real vector space $V$, nonnegativity on totally isotropic 2-planes implies that the Weitzenbock operator, $R_{2}$, on 2-forms (or 2-vectors) is positive semidefinite. If in addition condition $(*)$ is satisfied, then any element $\omega$ of the kernel of $R_{2}$ can be written in the form $\omega=\lambda \sum_{i=1}^{n} e_{2 i-1} \wedge e_{2 i}$ where $e_{1}, \ldots, e_{2 n}$ is an orthonormal basis of $V$ and $\lambda$ is a number. On a manifold satisfying the hypotheses of Theorem A, one takes a nonzero harmonic 2-form $\omega$, which must be parallel since $R_{2}$ is nonnegative at each point, and which at the point where $(*)$ holds is (up to a constant multiple) an orthogonal complex structure (for that tangent space). A short argument then shows that $\omega$ is in fact a globally defined orthogonal almost complex structure, which is integrable because it is parallel. Thus $M$ becomes a Kähler manifold. Knowing this, one proves that $b_{2}(M ; \mathbb{R})=1$ using the methods of [GK, §6]. One then computes that $c_{1}(M)$ (the first Chern class of $M$ ) is positive so, by Yau's resolution of the Calabi conjecture and a result of Kobayashi, $M$ is simply connected. Now that we know $M$ is Kähler, the proof of the first part of Theorem B is almost identical to that of Theorem 5 [GK].

The method for proving Theorem $\mathrm{B}(\mathrm{ii}), M$ is biholomorphic to $\mathbb{C P}^{n}$, is that of Siu and Yau in their resolution of the Frankel conjecture [SY]. This method is outlined in the wonderful book [W, pp. 523-527] (it was there that we first encountered that method). Very briefly, one must prove that any stable harmonic map $f: S^{2} \rightarrow M$ is \pm holomorphic and that if $f: S^{2} \rightarrow M$ is holomorphic then in the splitting of $f^{*} T M$ into $L_{1} \oplus \cdots \oplus L_{n}$ (where $T M$ is the holomorphic tangent bundle of $M$ and the $L_{i}$ are holomorphic line bundles over $\left.S^{2}\right)$ one has $c_{1}\left(L_{i}\right) \geq 1 \forall i$, and $c_{1}\left(L_{i_{0}}\right) \geq 2$ for some $i_{0}$. These last conditions yield $c_{1}\left(f^{*} T M\right)\left[S^{2}\right] \geq n+1$ and guarantee that the deformation theoretic arguments of $[S Y]$ work, proving that a positive generator of the free part of $H_{2}(M ; \mathbb{Z}) \cong \mathbb{Z}$ can be represented by a single holomorphic map $f: S^{2} \rightarrow M\left(H_{2}(M ; \mathbb{Z}) \cong \pi_{2}(M)\right.$ since $M$ is simply connected). We add that although the ideas for the proof of our theorems come from theorems concerning compact Kähler manifolds of positive holomorphic bisectional curvature, we do not actually use those theorems in our proofs.

We now address some linear algebraic questions concerning algebraic curvature tensors which arise in this paper. Suppose $R$ is an algebraic curvature tensor on a real inner product space $V($,$) and R$ has nonnegative curvature on totally isotropic 2-planes and satisfies $(*)$. The product curvature tensor on $\mathbb{R}^{n} \oplus \mathbb{R}^{1}$, which has constant curvature one on the $\mathbb{R}^{n}$ factor satisfies all these 
conditions. In fact given any two 2-planes $P$ and $Q$ in $\mathbb{R}^{n} \oplus \mathbb{R}^{1}$ such that $P \subset Q^{\perp}$ (cf. [Se]), one has $K(P)+K(Q) \geq 1$, so this curvature tensor satisfies $(*)$. Also, this curvature tensor has nonnegative curvature operator, so it has nonnegative curvature on totally isotropic 2-planes. Of course this is just the pointwise picture of $S^{n} \times S^{1}$, and shows that our Theorem A is false without the hypothesis $b_{2}(M ; \mathbb{R}) \neq 0$. Notice that the corresponding pointwise product curvature tensor for $S^{n} \times S^{2}$ does not satisfy $(*)$ but does have nonnegative curvature on totally isotropic 2-planes.

Our initial assumptions in Theorem $\mathrm{A}$, from a pointwise point of view, are that we are given an algebraic curvature tensor $R$ on an even-dimensional vector space $V$ which has nonnegative curvature on totally isotropic two planes and satisfies $(*)$. After we "globalize" this information via $b_{2}(M ; \mathbb{R}) \neq 0$, we get that in fact $R$ is Kähler. That is, there is an orthogonal complex structure $J$ on $V$ and $R$ satisfies (in addition to (a), (b), and (c) above) (d) $R(J X, J Y) Z=$ $R(X, Y) Z$ (equivalently $J(R(X, Y) Z)=R(X, Y)(J Z))$. If we now let $V$ be given an orthonormal basis of the form $e_{i}, J e_{i}, i=1, \ldots, n$, and let $e_{i *}=J e_{i} \forall i$, then $(*)$ yields, for the choices $e_{l}=e_{k^{*}}, e_{j}=e_{i^{*}}$,

$$
K_{i k}+K_{i k^{*}}>0 \text { for } i \neq k \text {. }
$$

Note that the sum in $(* *)$ is just the holomorphic bisectional curvature (cf. [GK] with the opposite sign convention for sectional curvature from ours) of the vectors $e_{i}, e_{k}$, sometimes written in the form $H\left(e_{i}, e_{k}\right)=\left(R\left(e_{i}, J e_{i}\right) J e_{k}, e_{k}\right)$ (perhaps $(* *)$ should be called "positive orthogonal holomorphic sectional curvature"; cf, [Se]). This shows that the canonical curvature tensor of the complex hyperquadric [KN, pp. 278-282] does not satisfy $(*)$ (as a real Riemannian manifold) since it does not satisfy $(* *)$. In fact, the canonical curvature tensor on the hyperquadric is "2-positive" in the terminology of [Si]. That is, for that curvature tensor, $\forall X$ (unit vector) $\exists Y$ such that $X, J X, Y$ are orthonormal and $H(X, Y)=0$ (and $Y$ and $J Y$ span the "null space" $H(X, \cdot)=0$ ). Of course the hyperquadric is a compact simply connected symmetric Kähler Einstein manifold, which shows the necessity of $(*)$ in arriving at the conclusions in Theorem B.

We now discuss ideas related to the \pm holomorphicity of stable harmonic maps $f: S^{2} \rightarrow M$.

Definition. Let $R$ be a Kähler algebraic curvature tensor on $V$ (with orthogonal complex structure $J)$. We say that $R$ has positive curvature on nondegenerate holomorphic isotropic 2-planes if for every isotropic $z \in V \otimes \mathbb{C},\langle\langle\widehat{R}(z \wedge J z)$, $z \wedge J z\rangle\rangle \geq 0$ with equality if and only if $z$ is an eigenvector for $J$ (so that the "plane" $z \wedge J z$ degenerates to 0 ).

Note that in the above definition $z$ and $J z$ automatically span a totally isotropic 2-plane if $z$ is not an eigenvector of $J$. If $R$ is a Kähler algebraic curvature tensor on $V$ satisfying (*) (or actually just $(* *)$ ), then $R$ has positive curvature on nondegenerate holomorphic isotropic 2-planes. This is because the plane spanned by $z$ and $J z$ is the same as that spanned by the $(1,0)$ and $(0,1)$ parts of $z, z^{\prime}$, and $z^{\prime \prime}$. If $z$ is isotropic, and not an eigenvector for $J$, we can find orthonormal $e_{i}, e_{k} \in V$ such that $\left(e_{i}, J e_{k}\right)=0$ and for which $\sqrt{2} z^{\prime} /\left\|z^{\prime}\right\|=e_{i}-l e_{i^{*}}$, and $\sqrt{2} z^{\prime \prime} /\left\|z^{\prime \prime}\right\|=e_{k}+l e_{k^{*}}$. We then get, 
using $z \wedge J z=-2 \imath z^{\prime} \wedge z^{\prime \prime}$,

$\langle\langle\widehat{R}(z \wedge J z), z \wedge J z\rangle\rangle=4\left\langle\left\langle\widehat{R}\left(z^{\prime} \wedge z^{\prime \prime}, z^{\prime} \wedge z^{\prime \prime}\right)\right\rangle\right\rangle=\left\|z^{\prime}\right\|^{2}\left\|z^{\prime \prime}\right\|^{2}\left(K_{i k}+K_{i k^{*}}\right)>0$

by $(* *)$. In the course of proving Theorem $\mathrm{B}$, we prove the following result, which may be of independent interest.

Lemma A. Let $(M, g, J)$ be a compact Kähler manifold which has nonnegative curvature on totally isotropic 2-planes and positive curvature on nondegenerate holomorphic isotropic 2-planes. Then every stable harmonic map $f: S^{2} \rightarrow M$ is \pm holomorphic.

Conditions like $(* *)$ show that our $(*)$ is related to the (algebraic) assumption that a Kähler curvature tensor has positive holomorphic bisectional curvature. While we do not have an example of an algebraic Kähler curvature tensor having property $(*)$ (and nonnegative curvature on totally isotropic 2-planes) but not having positive holomorphic bisectional curvature (nor an example of the opposite phenomenon) such an example should be constructable. As remarked previously, our arguments are logically independent of results proved for Kähler manifolds with positive holomorphic bisectional curvature, but the exact relation between that notion and $(*)$ is not entirely clear.

We add a word about notation. In general, our notation is the same as [KN]. This means in particular that the Kähler form, $\omega$, for $\mathbb{C}^{n}$ is $-l \sum d z_{i} \wedge d \bar{z}_{i}$ and in general the Kähler form for a Kähler manifold will be ilegative, where a positive $(1,1)$ form $\theta$ is a form which can be written $l \sum a_{i j} d z_{i} \wedge d \bar{z}_{j},\left(a_{i j}\right)$ a positive definite Hermitian matrix, or equivalently we can write $\theta(X, Y)=$ $a(J X, Y)$, where $a$ is a symmetric positive definite $J$-invariant bilinear form.

We would like to thank Olivier Debarre for many helpful discussions concerning various deformation theoretic and algebrogeometric aspects of the work of Siu and Yau.

Note added on April 15, 1991. This paper was completed in early November 1990. At the April 12-14, 1991 Seventh Annual Geometry Festival held at Duke University, the author spoke with Professor Mario Micallef concerning the results of the current paper. Professor Micallef knew the theorems in this paper already in 1987 , with the assumption $(*)$ replaced by the weaker assumption that $M$ be locally irreducible. Professor Micallef announced these results in the Mathematisches Forschungsinstitut Oberwolfach, Tagungsbericht 31/1987, Differentialgeometrie im Grossen, 19.7. bis 25.7.1987, pages 20-22. This author had no knowledge of that work by Professor Micallef. These results will be discussed in a forthcoming paper by M. Micallef and M. Wang.

\section{BACKground AND Theorem A}

Let $V$ be a real inner product space and $R$ an algebraic curvature on $V$ as in the introduction (so $R$ satisfies (a), (b), and (c)). The Ricci tensor, Ric, of $R$ is the symmetric bilinear form on $V$ defined by $\operatorname{Ric}(X, Y)=\sum\left(R\left(e_{i}, X\right) Y, e_{i}\right)$ where $e_{i}, i=1, \ldots, \operatorname{dim} V$, is an orthonormal basis for $V$ and $X, Y \in V$. The Wietzenbock operator of $R, R_{2}$ (cf. [Se]), may be defined as the map 
from 2-vectors to 2-vectors given by

$$
\begin{aligned}
\left(R_{2}\left(v_{1} \wedge v_{2}\right), w_{1} \wedge w_{2}\right)= & \operatorname{Ric}\left(v_{1}, w_{1}\right)\left(v_{2}, w_{2}\right)+\operatorname{Ric}\left(v_{2}, w_{2}\right)\left(v_{1}, w_{1}\right) \\
& -\operatorname{Ric}\left(v_{1}, w_{2}\right)\left(v_{2}, w_{1}\right)-\operatorname{Ric}\left(v_{2}, w_{1}\right)\left(v_{1}, w_{2}\right) \\
& -2\left(R\left(v_{1}, v_{2}\right) w_{2}, w_{1}\right)
\end{aligned}
$$

where $v_{1}, v_{2}, w_{1}, w_{2} \in V$ and the inner product $(, \quad)$ on $V$ is extended to $\bigwedge^{2} V$ as in the introduction. $R_{2}$ is extended to all of $\bigwedge^{2} V$ by linearity. $R_{2}$ turns out to be a symmetric operator. Of course, we may also consider $R_{2}$ as a map from 2-forms to 2-forms via ( , ), or as a $(4,0)$ tensor. If $X \in \wedge^{2} V$ the dual 2-form $\omega$ to $X$ is defined by $\omega(u, v)=(X, u \wedge v)$ and we can consider $X$ as a skew-symmetric linear map on $V$ by $(X(u), v)=(X, v \wedge u)$ so if $X=a \wedge b$, the $X(u)=(b, u) a-(a, u) b$. If $M$ is a Riemannian manifold with Riemannian metric $g$ and connection $\nabla$, then we define the Riemannian curvature tensor $R$, by

$$
\begin{array}{r}
R(X, Y) Z=\left(\nabla_{X} \nabla_{Y}-\nabla_{Y} \nabla_{X}-\nabla_{[X, Y]}\right) Z \\
X, Y, Z \text { vector fields on } M .
\end{array}
$$

At a point $p \in M$, for $X, Y \in T_{p} M$ spanning a plane $\sigma$, we define the sectional curvature of $\sigma, K(\sigma)$, by $K(\sigma)=g_{p}(R(X, Y) Y, X) /\|X \wedge Y\|^{2}$. If the context makes it clear we shall sometimes write $($,$) for g_{p}($,$) or g($,$) .$

The operator $R_{2}$ is the zeroth-order term appearing in the Weitzenbock formula for the Laplacian $\Delta$ acting on 2-forms on a Riemannian manifold (cf. [W]). If $\omega$ is a 2 -form on the Riemannian manifold $(M, g)$ then this formula can be written

$$
(\Delta \omega, \omega)=\frac{1}{2} \Delta\|\omega\|^{2}+\|\nabla \omega\|^{2}+\left(R_{2} \omega, \omega\right)
$$

For the record, one can use [W, WF.I, p. 304] to compute $\left(R_{2} \omega\right)\left(v_{1}, v_{2}\right)=$ $\omega\left(\operatorname{Ric} v_{1}, v_{2}\right)+\omega\left(v_{1}, \operatorname{Ric} v_{2}\right)-\sum \omega\left(R\left(v_{1}, v_{2}\right) e_{i}, e_{i}\right)$ (using (1.2) for the definition of $R$ ), and define $R_{2}$ on $\wedge^{2} V$ by $\omega\left(R_{2}\left(v_{1} \wedge v_{2}\right)\right)=R_{2} \omega\left(v_{1}, v_{2}\right)$; then using $\omega=$ the dual 2-form to the 2-vector $w_{1} \wedge w_{2}$ yields (1.1). On a compact oriented $M$, integrating (1.3) shows that if $R_{2}$ is a nonnegative operator at each point, then every harmonic 2 -form is parallel.

Proposition 1.1. Let $V$ and $R$ be as above and suppose $V$ is $2 n$-dimensional, $n \geq 2$. If $R$ has nonnegative curvature on totally isotropic 2-planes then $R_{2}$ is a nonnegative operator. If in addition $R$ satisfies $(*)$ then any $X$ in the kernel of $R_{2}$ may be written $X=\lambda \sum_{i=1}^{n} e_{2 i-1} \wedge e_{2 i}$ where $\lambda \in \mathbb{R}$ and $e_{1}, \ldots, e_{2 n}$ is an orthonormal basis of $V$.

Proof. Since $R_{2}$ is a symmetric operator on $\bigwedge^{2} V$ we can let $X \in V$ be an eigenvector of $R_{2}$, say $R_{2} X=r X, r \in \mathbb{R}$. There is an orthonormal basis $e_{1}, \ldots, e_{2 n}$ of $V$ and numbers $\lambda_{1} \geq \lambda_{2} \geq \cdots \geq \lambda_{n} \geq 0$ such that $X=\sum_{i=1}^{n} \lambda_{i} e_{2 i-1} \wedge e_{2 i}$ (this just amount to finding a "symplectic" basis for the dual 2-form, $\omega$, of $X$ ). There is no harm in assuming that $\lambda_{1}=1$. Direct 
computation using (1.1) now shows

$$
\begin{aligned}
& r=\left(r X, e_{1} \wedge e_{2}\right)=\left(R_{2}(X), e_{1} \wedge e_{2}\right)=\sum_{i=1}^{n} \lambda_{i}\left(R_{2}\left(e_{2 i-1} \wedge e_{2 i}\right), e_{1} \wedge e_{2}\right) \\
& =\left(R_{2}\left(e_{1} \wedge e_{2}\right), e_{1} \wedge e_{2}\right)+\sum_{i=2}^{n} \lambda_{i}\left(R_{2}\left(e_{2 i-l} \wedge e_{2 i}\right), e_{1} \wedge e_{2}\right) \\
& =\sum_{j=3}^{2 n}\left(K_{1 j}+K_{2 j}\right)-2 \sum_{i=2}^{n} \lambda_{i} R_{2 i-1,2 i, 2,1} \\
& =\sum_{l=2}^{n}\left\{K_{1,2 l}+K_{1,2 l-1}+K_{2,2 l}+K_{2,2 l-1}+2 \lambda_{l} R_{2,1,2 l, 2 l-1}\right\} \\
& \geq 2 \sum_{l=2}^{n}\left\{\left|R_{1,2,2 l, 2 l-1}\right|+\lambda_{1} R_{1,2,2 l, 2 l-1}\right\} \quad(\text { by }(0.2)) \\
& \text { (1.4ii) } \geq 2 \sum_{l=2}^{n}\left|R_{1,2,2 l, 2 l}\right|\left(1-\lambda_{1}\right) \geq 0 \text {. }
\end{aligned}
$$

Hence $R_{2}$ is a nonnegative operator. Now if $r=0$ then all of the above inequalities must be equalities. Therefore if $r=0$ we must have $K_{1,2 l}+$ $K_{2,2 l}+K_{1,2 l-1}+K_{2,2 l-1}=2\left|R_{1,2,2 l, 2 l-1}\right| \quad \forall l=2, \ldots, n$ (equality at (1.4i)) so if assumption (*) holds then this last $R$ term is nonzero. Because inequality (1.4ii) must also be an equality, we must have $\lambda=1 \quad \forall l$.

Note that any $X=\sum_{i=1}^{n} e_{2 i-1} \wedge e_{2 i} \quad\left(e_{k}\right.$ orthonormal $)$ is itself an orthogonal complex structure, $J$, on the vector space $V$, satisfying $J\left(e_{2 i-1}\right)=-e_{2 i}$. We now investigate algebraic Kähler curvature tensors.

Let $V$ be a $2 n$-dimensional $(n \geq 2)$ inner product space with an orthogonal complex structure $J$, and let $R$ be an algebraic Kähler curvature tensor on $V$ (so $R$ satisfies (d) in the introduction). Assume that $R$ has nonnegative curvature on totally isotropic 2-planes. Pick an orthonormal basis of $V$ of the form $e_{i}, J e_{i}, i=1, \ldots, n$, and let $e_{i^{*}}$ mean $J e_{i}$. Writing out (0.2) with $l=k^{*}, j=i^{*}$, and using the identity $R_{i i^{*} k^{*} k}=K_{i k}+K_{i k^{*}}$, we obtain

$$
K_{i k}+K_{i k^{*}} \geq 0 \text {. }
$$

As noted before, if $R$ also satisfies $(*)$, then the inequality in (1.5) is strict. Now write out $(0.2)$ with $k=i^{*}, l=j^{*}$, we obtain, after some simplification

$$
K_{i i^{*}}+K_{j j^{*}}+2 K_{i j^{*}} \geq 2\left|K_{i j}\right| \text {. }
$$

Writing out $(0.2)$ with $k=i^{*}$, replacing the original $j$ with $j^{*}$ and replacing the original $l$ with $j$, we obtain

$$
K_{i i^{*}}+K_{j j^{*}}+2 K_{i j} \geq 2\left|R_{i * j i j^{*}}\right|
$$

which simplifies to

$$
K_{i i^{*}}+K_{j j^{*}}+2 K_{i j} \geq 2\left|K_{i j^{*}}\right| .
$$

From (1.6) and (1.7) we deduce

$$
\forall i \neq j \quad K_{i i^{*}}+K_{j j^{*}} \geq 0 .
$$


It follows from identity (d) for $R$ that $\operatorname{Ric}(J X, J Y)=\operatorname{Ric}(X, Y)$. From this it follows that we may find an orthonormal basis of the above type, $e_{i}, J e_{i}$ "diagonalizing" Ric:

$$
\operatorname{Ric}\left(e_{i}, e_{j}\right)=\operatorname{Ric}\left(J e_{i}, J e_{j}\right)=\lambda_{i} \delta_{i j}, \quad \operatorname{Ric}\left(e_{i}, J e_{j}\right)=0,
$$

where $\lambda_{1} \geq \lambda_{2} \geq \cdots \geq \lambda_{n}$ are numbers. Now we compute

$$
\lambda_{i}=\operatorname{Ric}\left(e_{i}, e_{i}\right)=\sum_{a=1}^{n} R_{a^{*} a i i^{*}}=\sum_{a \neq i} R_{a^{*} a i i^{*}}+K_{i i^{*}}
$$

Hence,

$$
\lambda_{i}+\lambda_{j}=\sum_{a \neq i}\left(K_{a^{*} i}+K_{a i}\right)+\sum_{a \neq j}\left(K_{a^{*} j}+K_{a j}\right)+\left(K_{i i^{*}}+K_{j j^{*}}\right) .
$$

Since $R$ has nonnegative curvature on totally isotropic 2-planes, (1.5) implies that the first two summands on the right-hand side of (1.9) are nonnegative. Also, for $i \neq j,(1.8)$ implies that the third summand is nonnegative. If $R$ also satisfies $(*)$, then the first two summands on the right-hand side of (1.9) are strictly positive. This yields

(1.10) If $R$ is Kähler, with nonnegative curvature on totally isotropic 2planes then the "eigenvalues" $\lambda_{i}$ of Ric satisfy $\lambda_{i}+\lambda_{j} \geq 0$ for $i \neq j$. If $R$ satisfies $(*)$, then this inequality is strict. Thus the scalar curvature of $R$, $s=\operatorname{trace}(\mathrm{Ric})=2 \sum_{i=1}^{n} \lambda_{i}$ is nonnegative when $R$ has nonnegative curvature on totally isotropic 2-planes and strictly positive if $R$ also satisfies $(*)$.

This last remark follows from $\lambda_{1} \geq \cdots \geq \lambda_{n}$ and $\lambda_{n-1}+\lambda_{n} \geq 0 \quad(>0$ in the second case).

As above let $J$ be an orthogonal complex structure on $V$, which we write as $X=\sum e_{2 i-1} \wedge e_{2 i}$ when we want to consider $J$ as an element of $\wedge^{2} V$.

Proposition 1.2. Let $R$ be Kähler for $J$, have nonnegative curvature on totally isotropic 2-planes, and satisfy $(*)$. If $X=\sum e_{2 i-1} \wedge e_{2 i}\left(\right.$ where $\left.J\left(e_{2 i-1}\right)=-e_{2 i}\right)$ is in the kernel of $R_{2}$, then $\operatorname{ker}\left(R_{2}\right)=\mathbb{R} X$.

Proof. W extend $J$ to a linear map of $\wedge^{2} V$ by $J(u \wedge v)=J u \wedge J v$. Then on $\wedge^{2} V, J^{2}=1$, and every $Y \in \Lambda^{2} V$ can be written $Y=Y_{1}+Y_{2}$, where $J Y_{1}=Y_{1}, J Y_{2}=-Y_{2}$. It follows from the fact that $R$ is Kähler that if $Y \in \bigwedge^{2} V$ is in $\operatorname{ker}\left(R_{2}\right)$, then $Y_{i} \in \operatorname{ker}\left(R_{2}\right) i=1,2$ (i.e., $J R_{2}=R_{2} J$ on $\left.\wedge^{2} V\right)$. Also note that $J X=X$ since $X=\sum J e_{2 i} \wedge e_{2 i}$. We may assume that $e_{1}, \ldots, e_{2 n}$ diagonalizes Ric, since one can verify that for any orthonormal basis of the form $h_{i}, J h_{i}, X=\sum J h_{i} \wedge h_{i}$. Let $Y \in \operatorname{ker}\left(R_{2}\right)$, write $Y=Y_{1}+Y_{2}$ as above. Then we can write

$$
Y_{2}=\frac{1}{2} \sum_{i, j=1}^{n} a_{i j}\left(e_{i} \wedge e_{j}-J e_{i} \wedge J e_{j}\right)+c_{i j}\left(J e_{i} \wedge e_{j}+e_{i} \wedge J e_{j}\right)
$$

where the $a_{i j}, c_{i j}$ are skew-symmetric in $i, j$. A straightforward calculation now shows

$$
\left(R_{2} Y_{2}, Y_{2}\right)=2 \sum_{i \neq j}\left(a_{i j}^{2}+c_{i j}^{2}\right)\left(\lambda_{i}+\lambda_{j}\right)
$$

where the $\lambda_{i}, \lambda_{j}$ are the "eigenvalues" of Ric mentioned in (1.10). We see from (1.12) and (1.10) that if $(*)$ is satisfied and $Y_{2} \in \operatorname{ker}\left(R_{2}\right)$, then $Y_{2}=0$. 
We now consider $Y_{1}$ (cf. [GK, §6]). Define $T: V \rightarrow V$ by $(T u, v)=$ $\left(Y_{1}, J v \wedge u\right)$. Then $T$ is symmetric and commutes with $J$. We may therefore diagonalize $T$ with respect to an orthonormal basis of $V$ of the form $f_{i}, J f_{i}$ with $T f_{i}=\beta_{i} f_{i}, \beta_{1} \geq \beta_{2} \geq \cdots \geq \beta_{n}$, and we may assume $\beta_{1}>0$ if $Y_{1} \neq 0$. Thus $Y_{1}=\sum \beta_{i} J f_{i} \wedge f_{i}$. In the upcoming calculation, we use the fact that $\operatorname{Ric}(X, Y)=\sum_{i=1}^{n}\left(R\left(J h_{i}, h_{i}\right) X, J Y\right)$ where $h_{i}, J h_{i}$ is any " $J$-adapted" basis for $V$.

$$
\begin{aligned}
0 & =\left(R_{2} Y_{1}, f_{1^{*}} \wedge f_{1}\right)=\sum_{i=1}^{n} \beta_{i}\left(R_{2}\left(f_{i^{*}} \wedge f_{i}\right), f_{1^{*}} \wedge f_{1}\right) \\
& =\beta_{1} 2\left(\operatorname{Ric}\left(f_{1}, f_{1}\right)-K_{11^{*}}\right)-2 \sum_{i=2}^{n} \beta_{i} R_{i^{*} i 11^{*}} \\
& =\beta_{1} 2 \sum_{i=2}^{n} R_{i^{*} i 1^{*}}-2 \sum_{i=2}^{n} \beta_{i} R_{i^{*} i 1^{*}} .
\end{aligned}
$$

By $(* *), R_{i^{*} i 1^{*}}=K_{i 1}+K_{i 1^{*}}>0$ if $i \geq 2$, and $\beta_{1} \geq \cdots \geq \beta_{n}$, imply that (1.14) is nonnegative, so we get $\beta_{1}=\beta_{i} \forall i$. Hence, $Y_{1}=\beta_{1} \sum J f_{i} \wedge f_{i}$, so $\beta_{1} X=Y_{1}$.

Proof of Theorem A. Since $b_{2}(M ; \mathbb{R}) \neq 0$, by the Hodge and DeRham theorems, there is a nonzero harmonic 2 -form $\omega$ on $M$. Since $R$ has nonnegative curvature on totally isotropic 2-planes, $R_{2}$ is nonnegative at every point (Proposition 1.1) and (1.3) implies that $\omega$ is parallel and satisfies $R_{2} \omega=0$ (at every point). Let $p$ be the point where $(*)$ holds. By Proposition 1.1 we may assume that at $p$ we can write $X=\sum_{i=1}^{n} e_{2 i-1} \wedge e_{2 i}, e_{1}, \ldots, e_{2 n}$ an orthonormal basis for $T_{p} M$, where $X$ is the dual 2-vector for $\omega$, and we have multiplied $\omega$ by a constant ( $\omega$ has constant length since it is parallel). Then with $J$ defined by $J\left(e_{2 i-1}\right)=-e_{2 i}, J^{2}=-1$ (i.e., $X$ considered as a map on $T_{p} M$ ), we have

$$
\omega(u, v)=(u, J v) \text { for } u, v \in T_{p} M .
$$

We use equation (1.15) at every point of $M$ to define $J$ as a skew-symmetric endomorphism of $T M$. Then $J$ is parallel (as a section of $\operatorname{End}(T M)$ ) since $\omega$ and the metric are. Also $J^{2}$ is a parallel endomorphism of $T M$. Since $J_{p}^{2}=-\mathrm{id}_{T_{p} M}$ and since - id is a parallel endomorphism of $T M$, we get $J^{2}=-$ id everywhere on $M$. At any point $q \in M$ we can find an orthonormal basis $e_{1}, \ldots, e_{2 n}$ of $T_{q} M$ such that $J_{q}=\sum \gamma_{i} e_{2 i-1} \wedge e_{2 i}$. Then $J_{q}^{2}=-1$ implies that we can take each $\gamma_{i}=1$. Thus $J$ is an orthogonal almost complex structure on $M$ which is parallel, hence [KN, Theorem 4.3, p. 148] $J$ is integrable and endows $(M, g, J)$ with the structure of a Kähler manifold. This proves part (i) of Theorem A.

We now prove part (ii) of Theorem A. We now know that $M$ is Kähler, so that $R$ is Kähler. If $\eta$ is any harmonic 2-form on $M$ then $\eta$ is automatically parallel and hence satisfies $R_{2} \eta=0$ everywhere. At $p$, Proposition 1.2 implies $\eta=c \omega$, for some $c \in \mathbb{R}$, and by parallelism this must in fact be true at every point (with the same $c$ ). Thus every harmonic 2-form is a constant multiple of $\omega$, so $b_{2}(M ; \mathbb{R})=1$. Now the first Chern class of $M, c_{1}(M)$ is represented by the cohomology class of $(l / 2 \pi) \operatorname{tr} R$, where the trace is taken over $T^{1,0} M$ relative to the Hermitian extension of $(),,\langle\langle\rangle$,$\rangle . This yields$ 
$c_{1}(M)=[(1 / 2 \pi) \operatorname{Ric}(J \cdot, \cdot)]$. Since $b_{2}=1$, there is a constant $a$ such that

$$
a \omega=(1 / 2 \pi) \operatorname{Ric}(J \cdot, \cdot)+d \eta ; \quad \eta \text { a } 1 \text {-form, } \omega \text { the Kähler form }
$$

(actually it is known that we can write $d \eta=\partial \bar{\partial} F$ for a function $F$ ). Taking the inner product of both sides of (1.16) with $\omega$ and integrating over $M$ we obtain

$$
a=-(4 \pi n V)^{-1} \int_{M} s
$$

where $V=$ volume of $M, s=$ scalar curvature of $M$ (in our notation, $\left.\left(\omega, c_{1}\right)=-(4 \pi)^{-1} s\right)$. By $(1.10)$, the integral is strictly positive so $a<0$. Thus $c_{1}(M)$ is positive, that is we can represent $c_{1}(M)$ as $a(J$,$) where a$ is a positive definite symmetric bilinear form (in our case, $a=-a g$ ). By Yau's resolution of the Calabi conjecture [Y] we can find a Kähler metric on $M$ whose Ricci tensor is $-a g$, and being positive definite a result of Kobayashi [K] implies that $M$ is simply connected.

\section{THEOREM B}

Proof of Theorem B(i). The proof of Theorem 5 of [GK], which assumes $M$ is a compact Kähler-Einstein manifold of positive holomorphic bisectional curvature, does not invoke that positivity assumption until page 232 , line 12 , where positive holomorphic bisectional curvature is invoked to guarantee that, at the point $x$, where the holomorphic sectional curvature assumes its maximum, one has (in our notation) $R_{1^{*} 1 i i^{*}}>0$ for $i \geq 2$ where $e_{i}, e_{i^{*}}$ is an orthonormal basis of $T_{x} M$. This last $R$ term equals $K_{1 i}+K_{1 i^{*}}$ which, since $i \geq 2$, is positive in our case due to $(* *)$ (this is why we need to assume $(*)$ holds at every point of $M$ ). Also, $(* *)$ guarantees that, in the notation of [GK], $H_{1}=$ maximum value of the holomorphic sectional curvature, is positive, so the Einstein constant, $k$, is positive. The remainder of the proof of Theorem 5 of [GK] also does not use the assumption of positive holomorphic bisectional curvature, and thus completes the proof of our Theorem $\mathrm{B}(\mathrm{i})$.

Before the proof of Theorem B(ii), we do some preparatory work. We assume from now on that $M$ satisfies the hypotheses for Theorem B(ii), so that $M$ is a simply connected Kähler manifold with $b_{2}=1$, with nonnegative curvature on totally isotropic 2-planes, and satisfies $(*)$ (hence $(* *)$ ) at every point.

Proof of Lemma A (cf. [MM]). Let $f: S^{2} \rightarrow M$ be harmonic. Now $E=$ $f^{*}(T M \otimes \mathbb{C})$, given the induced Hermitian structure and connection from $M$, can be made into a holomorphic vector bundle over $S^{2}$ for which a section $W$ is holomorphic iff $\nabla^{0,1} W=0$. Consider $S^{2}$ as $\mathbb{C} \cup\{\infty\}$, and let $Z$ denote the holomorphic vector field which is $\partial / \partial z$ on $\mathbb{C}$ and 0 at $\{\infty\}$ (the 0 is of order 2). Then $f_{*} Z$ is a holomorphic, isotropic section of $E$. If $f$ is nontrivial, then this section can vanish for at most a finite number of points in $S^{2}$. In [MM, §5], the authors prove (cf. the proof of Theorem 3(i)) that for any compact manifold $M$ having nonnegative curvature on totally isotropic 2-planes, if $f$ is stable, then

$$
\left\langle\left\langle\widehat{R}\left(W \wedge\left(f_{*} Z\right)\right), W \wedge\left(f_{*} Z\right)\right\rangle\right\rangle=0
$$


at every point if $W$ is any holomorphic isotropic section of $E$ for which $f_{*} Z$ and $W$ span a totally isotropic 2-plane. In our setting, then we can take $W=J f_{*} Z$ (where $J$ is the parallel orthogonal almost complex structure on $E$ induced from the $J$ on $T M$ ) which is holomorphic because $\nabla_{\partial / \partial \bar{z}}\left(J f_{*} Z\right)=$ $J\left(\nabla_{\partial / \partial \bar{z}}\left(f_{*} Z\right)\right)=0$, isotropic because $f_{*} Z$ is, and $f_{*} Z$ and $J f_{*} Z$ span a totally isotropic 2-plane since $\left(f_{*} Z, J f_{*} Z\right)=0$. Since our $M$ has nonnegative curvature on totally isotropic 2-planes and positive curvature on nondegenerate holomorphic isotropic 2-planes, (2.1) implies that $J f_{*} Z= \pm l f_{*} Z$ on an open set, i.e., $f$ is \pm holomorphic on an open set. But a harmonic map which is \pm holomorphic on an open set is \pm holomorphic [EL, Theorem (9.4), p. 53]. Hence $f$ is \pm holomorphic.

Suppose now $f: S^{2} \rightarrow M$ is a nonconstant holomorphic map. Let $T M$ be the holomorphic tangent bundle of $M$. A theorem of Grothendieck [G, Theorem 2.1, p. 126] guarantees that the holomorphic vector bundle $f^{*} T M$ splits holomorphically into a direct sum $L_{1} \oplus \cdots \oplus L_{n}$ of holomorphic line bundles.

We now claim

$$
c_{1}\left(L_{i}\right) \geq 1 \forall i \text { and } c_{1}\left(L_{i_{0}}\right) \geq 2 \text { for some } i_{0} .
$$

In (2.2) we have written $c_{1}\left(L_{i}\right)$ for $c_{1}\left(L_{i}\right)\left[S^{2}\right]=\int_{S^{2}} c_{1}\left(L_{i}\right)$. Actually, the proof of the second part of $(2.2)$ is immediate [F, p. 115]: the holomorphic section of $f^{*} T M, f_{*} Z$, projects to a holomorphic section on each $L_{i}$, which must be nontrivial for some $i_{0} ; f_{*} Z$ has a zero of order two at $\infty$ and no poles, thus, as a section of $L_{i_{0}}, f_{*} Z$ has a zero of order two and no poles whence $c_{1}\left(L_{i_{0}}\right)=$ sum of zeros minus poles (with multiplicities) of any meromorphic section, must be at least two.

To prove the first part of $(2.2)$, let $B=$ the dual bundle to $f^{*} T M$, given the dual Hermitian metric and connection. Let $S=$ the dual bundle to one of the $L_{i}$. We prove that $c_{1}\left(L_{i}\right)>0$ by proving $c_{1}(S)<0$, and we prove that by contradiction (cf. [Si, p. 648, last paragraph] and [B, p. 287]). If $c_{1}(S) \geq 0$, then by the Riemann-Roch Theorem, there is a nontrivial holomorphic section $s$ of $S$. We can consider $s$ as a holomorphic section of $B$, and let $Y$ be the metric dual to $s$ given by $\langle\langle X, Y\rangle\rangle=s(X) \quad \forall X \in f^{*} T M$. A computation using the second fundamental form, $A$, of $S$ in $B$ (cf. [GH, pp. 78, 79]) yields

$$
\left\langle\left\langle R^{B}(X, \bar{X}) s, s\right\rangle\right\rangle=\left\langle\left\langle R^{S}(X, \bar{X}) s, s\right\rangle\right\rangle+\|A(X, s)\|^{2}
$$

for any holomorphic vector field $X$ on $S^{2}$.

Using the dual, $Y,(2.3)$ can be written

$$
-\langle\langle Y, R(X, \bar{X}) Y\rangle\rangle=\left\langle\left\langle R^{S}(X, \bar{X}) s, s\right\rangle\right\rangle+\|A(X, s)\|^{2},
$$

where $R=$ the curvature tensor on $E$.

Since $f_{*} Z$ has a zero at $\infty$ and is holomorphic, we get $s\left(f_{*} Z\right)=0$ at each point, so $\left\langle\left\langle f_{*} Z, Y\right\rangle\right\rangle=0$ at each point. Since $Y$ is a section of $f^{*} T M$ where $T M$ is the holomorphic tangent bundle, we may write $Y=A-l J A$, for some secton of $f^{*} T M$ where now $T M$ refers to the real tangent bundle to $M$ (i.e., we can write $Y_{p}=A_{f(p)}-l J A_{f(p)}$ where $\left.A \in T_{f(p)} M \quad \forall p \in S^{2}\right)$, and writing $f_{x}$ for $f_{*}(\partial / \partial x)$, we get $\left\langle\left\langle f_{x}, A\right\rangle\right\rangle=\left\langle\left\langle f_{x}, J A\right\rangle\right\rangle=0$. On the other hand, one computes that $-\langle\langle Y, R(X, \bar{X}) Y\rangle\rangle$, with $X=Z$, equals $-4\left(R\left(f_{x}, J f_{X}\right) J A, A\right)$. 
This last term is strictly negative by $(* *)$, except at the finite number of points where $Y$ or $f_{*} Z$ vanishes. Thus the left-hand side of (2.4) is negative almost everywhere so

$$
0>\left\langle\left\langle R^{S}(Z, \bar{Z}) s, s\right\rangle\right\rangle=R^{S}(Z, \bar{Z})\|s\|^{2}=4 \pi c_{1}(S)(X, J X)\|s\|^{2}
$$

(where $X=\operatorname{Re} Z$ ) almost everywhere, and this yields $c_{1}(S)<0$, which is a contradiction. Hence $c_{1}\left(L_{i}\right)>0 \quad \forall i$.

Proof of Theorem B(i) (cf. [SY, Si, F, W]). By Theorem A, $H^{2}(M ; \mathbb{Z}) \cong \mathbb{Z}$, $H_{2}(M ; \mathbb{Z}) \cong \mathbb{Z} \oplus$ torsion, and $\pi_{2}(M) \cong H_{2}(M ; \mathbb{Z})$. Choose a generator $a$ of $H^{2}(M ; \mathbb{Z})$ which is a negative multiple of the (class of the) Kähler form $\omega$ (so $a$ is positive), and let $g$ be a generator of the free part of $\pi_{2}(M)$ such that $a(g)=1$. By a result of Kobayashi and Ochiai [KO, Corollary to Theorem 1.1 , p. 46], we will be done if $c_{1}(M)(g) \geq n+1$. We may represent the free homotopy class of $g$ by $\sum_{i=1}^{k} f_{i}$ where each $f_{i}$ is harmonic and energy minimizing in its free homotopy class [SU, Theorem 5.5, p. 22]. By Lemma A, each $f_{i}$ is \pm holomorphic. By (2.2), if $k=1$ and $f_{1}$ is holomorphic, we have $c_{1}(M)(g)=c_{1}(M)\left[f_{1}\left(S^{2}\right)\right]=c_{1}\left(f_{1}^{*}(T M)\right)\left[S^{2}\right] \geq n+1$. Note that if $k=1$, then since $c_{1}(M)$ is a positive multiple of (the class of) $-\omega, f_{1}$ must be holomorphic. Thus we are reduced to showing that $k=1$. This means we want to show that Proposition 3, p. 201 of [SY] holds under our hypotheses, for if that proposition does hold, then the proof that $k=1$ is identical to that of Claim 2 of [SY, pp. 202-203]. As was already observed by Futaki [F, Claim 1, p. 119] the cited Proposition of [SY] remains true as long as $f^{*} T M$ splits into a direct sum of positive line bundles, where $f: S^{2} \rightarrow M$ is holomorphic (such an $f$ is called "type I" in [F]). Briefly, in the notation of [SY, p. 197], each $L_{i}$ positive (actually nonnegative is sufficient) guarantees that the deformation map $F: D \times V \rightarrow S^{2} \times M$ is a submersion at $(d, p)$, where $d=$ the point of $D$ corresponding to $V\left(V\right.$ is the graph of $f$ in $\left.S^{2} \times M\right)$ and $p$ is a point of $S^{2}$ where $f$ is an immersion $\left(c_{1}\left(L_{i}\right) \geq 0 \quad \forall i\right.$ implies that $H^{1}\left(V, N_{V}\right)=0$, where $N_{V}$ is the normal bundle to $V$, and guarantees that $N_{V}$ is generated by global holomorphic sections at each point). Thus $F$ is onto, which shows that one can find a deformation of $V$ passing through any point of $S^{2} \times M$ (although this deformed $V$ might not be the graph of a function $S^{2} \rightarrow M$ ). Now blowing up at a given point of $V$ and repeating the previous argument (which goes through because blowing up at one point decreases the $c_{1}$ 's by one, so they are still nonnegative) shows that any tangent direction can be chosen for a deformation through that point. Thus our (2.2) is enough to guarantee that Proposition 3 of [SY] does hold, so we are done.

\section{BIBLIOGRAPHY}

[B] S. Bando, On the classification of three-dimensional compact Kaehler manifolds of nonnegative bisectional curvature, J. Differential Geom. 19 (1984), 283-297.

[EL] J. Eells and L. Lemaire, Selected topics in harmonic maps, CBMS Regional Conf. Ser. in Math., vol. 50, Amer. Math. Soc., Providence, R.I., 1985.

[F] A. Futaki, On compact Kähler manifolds with semi-positive bisectional curvature, J. Fac. Sci. Univ. Tokyo Sect. 1A Math. 28 (1981), 111-125.

[GH] P. A. Griffiths and J. Harris, Principles of algebraic geometry, Wiley, New York, 1978. 
[G] A. Grothendieck, Sur la classification des fibres holomorphes sur la sphere de Riemann, Amer. J. Math. 79 (1957), 121-138.

[GK] S. I. Goldberg and S. Kobayashi, Holomorphic bisectional curvature, J. Differential Geom. 1 (1967), 225-233.

[K] S. Kobayashi, On compact Kähler manifolds with positive Ricci tensor, Ann. of Math. (2) 74 (1961), 570-574.

[KN] S. Kobayashi and K. Nomizu, Foundations of differential geometry. II, Wiley-Interscience, New York, 1969.

[KO] S. Kobayashi and T. Ochiai, Characterization of complex projective spaces and hyperquadrics, J. Math. Kyoto Univ. 13 (1973), 31-47.

[MM] M. Micallef and J. Moore, Minimal two-spheres and the topology of manifolds with positive curvature on totally isotropic two-planes, Ann. of Math. (2) 127 (1988), 199-227.

[SU] J. Sacks and K. Uhlenbeck, The existence of minimal immersion of 2-spheres, Ann. of Math. (2) 113 (1981), 1-24.

[Se] W. Seaman, Orthogonally pinched curvature tensors and applications, Math. Scand. 69 (1991), 5-14.

[Si] Y.-T. Siu, Curvature characterization of hyperquadrics, Duke Math. J. 47 (1980), 641-654.

[SY] Y.-T. Siu and S.-T. Yau, Compact Kähler manifolds of positive bisectional curvature, Invent. Math. 59 (1980), 189-204

[W] H. Wu, The Bochner technique in differential geometry, Math. Rep., vol. 3, Harwood Academic, London, 1988, pp. 289-538.

[Y] S.-T. Yau, On the Ricci curvature of a compact Kähler manifold and the complex MongeAmpere equation. I, Comm. Pure Appl. Math. 31 (1978), 339-411.

Department of Mathematics, University of Iowa, Iowa City, Iowa 52242

E-mail address: walter-seaman@uiowa.edu 